\title{
Opinion
}

Copyright (C) All rights are reserved by Alma Miric

\section{Brain Homeostasis and Aspartate Levels in Maintaining of Brain's Functioning}

\author{
Alma Miric* \\ Department of Molecular Biology and Physiology, Belgrade University, Serbia
}

*Corresponding author: Alma Miric, Department of Molecular Biology and Physiology, Belgrade University, Serbia.

\section{Opinion}

Brain and its optimal function are depending on many factors, such as nutrition, sleep, sexual activity, lifestyle-drinking habits, drugs, meditation, yoga, and homme per homme interactions. Studying brain is complex and interdisciplinary skill, and many hypotheses were proposed such as modular theory of brainsociobiology" Sober". Many neurotransmitters are involved in function of brain, such as serotonin, dopamine, amines, glutamate, aspartate, GABA.

Key neurotransmitters in brain functioning are glutamate, GABA precursor, and aspartate. Low concentrations of each of them are crucial for brain homeostasis balance. Glutamate receptors NMDA which bind glutamate and other substances, have various effects on relaxing brain state, especially alfa state during sleep. Competition between GABA and aspartate for NMDA receptor is normal, as antagonists, but in states like nervous breakdown , sleep deprivation, stress, involuntary movements of hands and legs, and rotation of eyes, as a consequence of disturbance in simpaticus/parasimpaticus functioning and homeostasis, also a sleep deprivation are symptoms of alteration in aspartate levels. Aspartate levels are higher than usual, and this finding is found experimentally true. Many experiments were conducted where aspartate levels were added into spine of patients via injection. Such intervention caused involuntary movements.

Dopamine also have impact on brain functioning, especially on involuntary movements. As additional effect.

\section{NMDA Receptor Antagonists}

GABA and aspartate are antagonists. Higher levels of glutamate then aspartate is found in population of people with normal sleeping patterns. When these two neurotransmitters are competing for binding place on NMDA receptors, in states like nervous breakdown there are higher concentrations of aspartate and involuntary movements are usual. Patients can't drive bicycle or car or can't sleep.

\section{Acknowledgement}

None.

\section{Conflict of Interest}

No conflict of interest. 